\title{
EL FRACASO EN LA RESOLUCIÓN DE PROBLEMAS DE FÍSICA: UNA INVESTIGACIÓN ORIENTADA POR NUEVOS SUPUESTOS
}

\author{
GIL PÉREZ, D., MARTÍNEZ TORREGRosa, J. y SENENT PÉREZ, F. \\ Universitat Autònoma de Barcelona y Universitat de València.
}

II Congreso Internacional sobre investigación en la didáctica de las Ciencias y las Matemáticas. Valencia, 1987.

\section{SUMMARY}

This paper shows an attempt to question the usual way of teaching problem solving and to elaborate more effective theoretically based proposats. Some results of the applications of new orientations are included.

\section{INTRODUCCIÓN}

La resolución de problemas de Física y, en general, de ciencias y matemáticas, ha figurado durante decenios, como muestra una abundante literatura (Garrett, 1986), entre las lineas de investigación prioritarias, debido, en gran parte, a las elevadas tasas de fracaso de los alumnos en dicha tarea (Martinez Torregrosa, 1987). Sin embargo, este constante interés, que sigue actualmente vigente (Garrett, 1987, Mahapatra, 1987), no ha producido mejoras sensibles en los resultados obtenidos por los alumnos. En nuestra opinión ello podría ser debido a las orientaciones y supuestos básicos de la investigación realizada hasta aquí y obligaria a concebir nuevos enfoques. En efecto, si, como dijo Bachelard, «todo conocimiento es la respuesta a una cuestión", las preguntas que nos formulemos van a determinar en gran medida la validez de la investigación realizada. Sin embargo, en la investigación didáctica, tanto o más que en otros campos, las preguntas a las que responde la investigación están cargadas de significado implicito que rara vez es sometido a análisis, escapando asi a la crítica. De este modo, resulta posible que toda una linea de investigación quede prisionera durante años de unas determinadas preguntas que, lo menos que cabe decir es que no son las únicas posibles.

Hacer avanzar la investigación demanda una cuidadosa atención a dichas cuestiones y un esfuerzo por romper la limitación que suponen las preguntas más obvias. El trabajo que aquí presentamos responde a dicho intento por abordar de forma crítica la investigación sobre el fracaso en la resolución de problemas de Física.

\section{LOS SUPUESTOS IMPLICITOS EN LA IN- VESTIGACIÓN SOBRE RESOLUCIÓN DE PROBLEMAS}

Si analizamos la literatura existente sobre resolución de problemas podemos constatar que una buena parte de esa investigación ha estado dedicada a mostrar las diferencias entre los expertos y principiantes o, más en general, entre los buenos resolventes y los mediocres (Larkin y Reif, 1979; Finegold y Mass, 1985), con objeto de establecer las características de una buena resolución y extraer recomendaciones útiles para los alumnos.

Como ya hemos señalado, Ia abundante investigación realizada con dicha orientación, aunque ha producido, sin duda, algunos resultados de interés, no ha contribuido a reducir las elevadas tasas de fracaso de los alumnos. Conviene, pues, explicitar los supuestos a que responde esa investigacion, con objeto de concebir otros posibles enfoques.

Un primer supuesto que subyace claramente en la pregunta « ¿Qué diferencia un buen resolvente de otro mediocre?» es el de una responsabilidad individual (hay quien resuelve bien los problemas y hay quien no). Ello es coherente con las explicaciones que el profesorado suele dar acerca del fracaso en esta tarea. En efecto, cuando se pregunta al profesorado en activo cuáles pueden ser las causas del fracaso generalizado en la resolución de problemas de Física, las únicas razones apuntadas por un porcentaje significativo de los encuestados son (Gil y Martínez Torregrosa, 1984):

- falta de suficientes conocimientos teóricos 
- escaso dominio del aparato matemático

- lectura no comprensiva del enunciado.

Se trata, como puede verse, de explicaciones que atribuyen el fracaso a las carencias de los alumnos, lo que sin duda constituye una de las ideas clave en el «pensamiento espontáneo» de los profesores (Bromme, 1984). Pero, ¿cómo puede aceptarse este tipo de razones cuando el fracaso afecta a la mayoría de los alumnos?

La investigación realizada en torno a la pregunta "¿Qué diferencia un buen de un mal resolvente?" responde, además, a un segundo supuesto: el de que es posible extraer unas recomendaciones de cómo resolver bien los problemas y transmitirlas a los alumnos como forma cfcctiva de ayuda. Este mismo supuesto subyace en otro gran bloque de la investigación realizada que, desde las clásicas propuestas de Polya (1957), ha estado dirigida hacia la elaboración de modelos de resolición $\mathrm{e}$ incluso de algoritmos más o menos precisos (Dumas-Carré, 1987). Se trata de un supuesto coherente con el paradigma de enseñanza/aprendizaje por transmisión/recepción de conocimientos ya elaborados, cuya ineficacia ha sido reiteradamente constatada y denunciada (Gil, 1983; Millar y Driver, 1987).

En definitiva, la pregunta sobre las diferencias entre buenos y malos resolventes aparece como expresión de unos determinados supuestos que deben ser considerados meras hipotesis y no como algo incuestionable, evitando así que dirijan la investigación en una única dirección, en ocasiones escasamente fructífera. Y son necesarias nuevas preguntas que (re)orienten la investigación.

Es posible asi preguntarse ¿qué es lo que los profesores hacemos para enseñar a resolver problemas? o, más precisamente, ¿qué es lo que hacemos para que la mayoría de los alumnos sea incapaz de enfrentarse a un problema en cuanto se aleja minimamente de los que han resuclto en clase?

Una pregunta como la anterior dirige la atención sobre las orientaciones didácticas de profesores y textos y responde a otra hipótesis: la de que un resultado de fracaso generalizado como el que se da en la resolución de problemas tiene su origen en errores de planteamiento en el proceso de enseñanza/aprendizaje. $Y$ aún es posible bucear más en profundidad y preguntar ¿qué ha de entenderse por problema? y ¿en qué medida lo que se enseña en las clases se aproxima a una auténtica resolución de problemas? Se trata de cuestiones que dirigen la atención sobre las actividades del profesorado y en las que subyace el supuesto de la importancia crucial de las actividades, actitudes y expectativas del profesorado en la efectividad del aprendizaje, puesta en evidencia, a lo largo de las dos últimas décadas por la denominada effective school research (Ribas, 1986). Nuestro trabajo responde a estas últimas cuestiones, más aún, responde a un intento de mostrar que es posible cambiar esta situacion, modificar las actividades, actitudes y expectativas del profesorado a partir de un planteamiento en profundidad de las cuestiones anteriores por los propios profesores, si se facilita un trabajo colectivo de «reflexión descondicionadas, es decir, capaz de romper las limitaciones que suponen las preguntas y supuestos más obvios, de los que a menudo quedamos prisioneros.

Describiremos, a continuación, el proceso seguido en un curso sobre resolución de problemas dirigido a profesores en activo y en formación planteado como sesiones de trabajo para unos 40 profesores (númcro similar al de los alumnos de un aula) estructurados en pequeños grupos. Intentaremos mostrar así que dicho trabajo hace posible la puesta en cuestión de la didáctica habitual de resolución de problemas y la elaboración fundamentada de propuestas más efectivas.

\section{ACTIVWADES DE TOMA DE CONCIENCIA}

Como ya hemos indicado, cuando se pregunta al profesorado en activo cuáles pueden ser las causas del fracaso generalizado en la resolución de problemas de Física, raramente se aducen razones que inculpen a la propia didáctica empleada. Conviene, pues, que una de las primeras actividades a realizar en el curso conduzca, precisamente, a poner en cuestión dicha didáctica, a hacer sentir «en carne propia» las deficiencias de la enseñanza habitual de la resolución de problemas. Basta, para ello, proponer algún pequeño ejercicio, aparentemente muy sencillo, pero que conduzca, a consecuencia del operativismo mecánico habitual, a resultados totalmente erroneos. Un ejemplo de tales ejcrcicios que se ha utilizado reiteradamente con éxito (Gil, 1982) es el siguiente:

Calcular la distancia recorrida en $5 \mathrm{~s}$ por un objeto que se desplaza a lo largo de su trayectoria según la ecuación: $\mathrm{e}=25+40 \mathrm{t}-5 \mathrm{t}^{2}$ (e en $\mathrm{m}$ si $\mathrm{t}$ en $\mathrm{s}$ ).

La casi totalidad de los asistentes «resuelve» muy rápidamente el ejercicio, dando como respuesta, en general, $100 \mathrm{~m}$ o $75 \mathrm{~m}$. Sin entrar en la discusion de esta discrepancia, proponemos que calculen la distancia recorrida por el mismo móvil en 6 segundos. Los resultados obtenidos ahora $(85 \mathrm{~m}$ quienes antes obtuvieron $100 \mathrm{~m}$ y $60 \mathrm{~m}$ quienes obtuvieron $75 \mathrm{~m}$ ) muestran claramente que "algo va mal» (iel móvil no puede haber recorrido en más tiempo menos distancia!). Estos son los resultados obtenidos habitualmente por los alumnos y también, repetimos, por muchos profesores. $\mathrm{La}$ resolución de este aparente enigma es, por supuesto, sencilla y, tras una pequeña reflexión, los asistentes ( $y$ también los alumnos en sus clases) comprenden que la ecuación $\mathrm{e}=25+40 \mathrm{t}-5 \mathrm{t}^{2}$, corresponde al movimiento de un objeto que avanza con velocidad decreciente hasta pararse y comenzar a retroceder. Obtienen así los resultados correctos, que son $85 \mathrm{~m}$ a los 5 s y $100 \mathrm{~m}$ a los $6 \mathrm{~s}$. 
Pero lo que nos interesa aqui es reflexionar sobre el hecho de que un problema tan sencillo conduzca a resultados erróneos de forma muy generalizada. Conviene, pues, proceder a una reflexión/discusión en tomo a:

¿Por qué se dan unos resultados erróneos tan generalizados en un problema como el anterior? ¿De qué pueden ser índice? ¿Qué sugieren?

Los resultados del ejercicio que acabamos de comentar actúan de utoma de conciencia» y conducen a un debate detenido, que pone en cuestion la actividad del propio profesorado. Se hace referencia así, entre otras, a las siguientes características de la orientación dada habitualmente a la resolución de problemas:

- La falta de reflexión cualitativa previa, o, dicho de otro modo, el operativismo mecánico con que se abordan habitualmente los problemas, incliso por los mismos profesores. Conviene recordar a este respecto las palabras de Einstein: «Ningún científico piensa con fórmulas. Antes que el físico comience a calcular debe tener en su cerebro el curso de los razonamientos. Estos últimos, en la mayoría de los casos, pueden ser expuestos con palabras sencillas. Los cálculos y las fórmulas constituyen el paso siguiente». Sin embargo, insistimos, la didáctica habitual de resolución de problemas suele impulsar a un operativismo abstracto, carente de significado, que poco puede contribuir a un aprendizaje significativo.

- Un tratamiento superficial que no se detiene en la clarifícación de los conceptos. Así, en el problema considerado, se producen evidentes confusiones entre distancia al origen, desplazamiento y distancia recorrida. Y no se trata de una cuestión puramente terminológica de escasa importancia, sino índice, repetimos, de un tratamiento superficial que en poco puede favorecer una auténtica comprensión de los conceptos. Más aún: se manejan casi exclusivamente situaciones que favorecen las confusiones. En el caso que nos ocupa, por ejemplo, la mayor parte de los problemas sobre móviles, toman como sistema de referencia (explícita, o, más a menudo, implicitamente) el punto e instante en que el movimiento se inicia, y sentido positivo el del movimiento, con lo cual el espacio $e$ (distancia al origen) coincide con el desplazamiento $\triangle e$; si además no hay retrocesos, el valor de la distancia recorrida coincide también. La repetición de ejemplos en que esto ocurre lieva, no sólo a confundir los conceptos, sino incluso a hacer «innecesaria» la atención al sistema de referencia. El carácter relativo de todo movimiento es asi escamoteado, negado en la práctica, por mucho que se haya insistido en él teóricamente. $Y$ es necesario tener presente que esta costumbre a absolutivizar el movimiento, tomando siempre como referencia implícita el punto e instante de donde parte el móvil, corresponde a tendencias profundamente arraigadas en el nitto a centrar todo el estudio en símismo, en su propia experiencia, generalizándola acríticamente (Piaget, 1974).
De este modo, los problemas, en vez de contribuir a un aprendizaje significativo, conectando con los preconceptos de los alumnos y ayudando a romper con visiones confusas, favorecen su afianzamiento. Y esto ocurre incluso o, mejor sobre todo, cuando se llega a resultados correctos. Pensemos en los numerosos problemas sobre caída de graves que se realizan y que los alumnos llegan a hacer casi con los ojos cerrados; ello no impide que sigan pensando que «un cuerpo de doble masa caerá en la mitad de tiempo». Es decir, los problemas «correctamente» resueltos no han permitido poner en cuestión el preconcepto de la influencia de la masa.

En resumen, los problemas, en vez de ocasión privilegiada para construir y profundizar los conocimientos, se convierten en refuerzo de errores conceptuales y metodologicos. Podría pensarse que hay mucha exageración en estas conclusiones; pero basta referirse a los análisis realizados sobre los problemas resueltos en los textos o por profesores (Gil y Martínez Torregrosa, 1984), para constatar que el operativismo, el tratamiento superficial - sin siquiera análisis de los resultadoses realmente muy general entre el mismo profesorado (véase el anexo I).

La discusión anterior motiva, pues, una «toma de conciencia» que hace que los propios profesores apunten claramente deficiencias que se separan de las causas de fracaso generalmente aceptadas. Son estas deficiencias -señaladas, repetimos, por los propios profesoreslas que conducen a la necesidad de un replanteamiento en profundidad de la didáctica habitual de la resolución de problemas.

\section{NECESIDAD DE UN REPLANTEAMIENTO EN PROFUNDIDAD}

Las mayores dificultades que con frecuencia ha encontrado el desarrollo de una ciencia han derivado de supuestos implicitos, aceptadas sin cuestionamiento alguno, que escapan asi a la crítica. En tales casos se impone, como la historia de las ciencias ha mostrado reiteradamente, un replanteamiento en profundidad que analice criticamente hasta lo más obvio. Por lo que se refiere a la didáctica de la resolución de problemas, ello supone descender hasta la clarificación misma de la idea de problema. Esta es, pues, la actividad que proponemos a los grupos de trabajo:

\section{¿Qué hemos de entender por problema?}

Los resultados de la discusión coinciden en caracterizar como problema las situaciones que plantean dificultades para las que no se poseen soluciones hechas. Se trata, por supuesto, de una idea justa, básicamente aceptada por quienes han abordado explícitamente la cuestion (Hudgins, 1966; Riche, 1978; Mettes et Al., 1980; Hayes, 1981; Bodner y McMillen, 1986). Pero 
lo importante es relacionar esta idea de lo que es un problema con lo que se hace en clase:

\section{¿En qué medida las explicaciones de los problemas he- chas por los profesores o expuestas en los libros de texto están de acuerdo con su naturaleza de tarea descono- cida, para la que de entrada no se posee solución?}

La discusión generada por esta actividad pone totalmente en cuestión la práctica docente habitual; se senala, en efecto, que los "problemas» son explicados como algo que se sabe hacer, como algo cuya solución se conoce y que no genera dudas ni exige tentativas: el profesor conoce la situación - para él no es un problema- y la explica linealmente, «con toda claridad", consecuentemente, los alumnos pueden aprender dicha solución y repetirla ante situaciones idénticas, pero no aprenden a abordar un verdadero problema y cualquier pequeño cambio les supone dificultades insuperables, provocando el abandono. En definitiva, esta discusión en torno a qué entender por problema permite realizar una crítica más profunda de la didáctica habitual. Puede ahora darse un paso más y plantear:

Si un problema es una situación para la que no se tiene respuesta elaborada, ¿cómo habrá que enfocar su resolución?

Las propuestas de los grupos coinciden básicamente en señalar la necesidad de una orientación que se aproxime a lo que es el tratamiento científico de verdaderos problemas, es decir, que se aproxime a lo que entendemos por metodología científica. Ello obliga a un esfuerzo de clarificación al respecto, que evite visiones simplistas e incorrectas - muy extendidas entre el profesorado (Gil, 1983; Hodson, 1985; Millar y Driver, 1987) -, que colocan la "observación» en el origen de toda investigación y olvidan el papel del pensamiento divergente, - de la invención de soluciones a titulo de hipotesis- como núcleo central del trabajo científico (Gi1, 1983 y 1986). Es esta una discusión que puede exigir más o menos tiempo en función del peso que las habituales visiones inductivistas tengan entre los asistentes. En cualquier caso, se trata de una clarificación absolutamente necesaria, no sólo para un correcto planteamicnto de la resolución de problemas, sino para hacer posible una visión menos simplista de lo que es la ciencia. Y de nuevo ahora, esta clarificación permite una critica más profunda, menos condicionada, de cómo se plantea habitualmente la resolución de problemas:

\section{¿Qué es lo que en los enunciados habituales dificulta un tratamiento científico de los problemas y deja, en particular, sin sentido la tarea fundamental de emisión de hipótesis?}

El paso a dar ahora no es, ciertamente, fácil; pero el hilo conductor seguido hasta aquí permite que algu- nos grupos lleguen a ver que la inclusión de los datos en el enunciado, respondiendo a concepciones inductivistas, orienta la resolución hacia el manejo de unas determinadas magnitudes sin que ello responda a una reflexion cualitativa ni a las subsiguientes hipótesis. De este modo, al abordar un problema, el alumno se ve abocado a buscar aquellas ecuaciones que pongan en relación los datos e incógnitas proporcionados por el enunciado, cayendo así en un puro operativismo. No basta, pues, denunciar dicho operativismo: se trata de hacerlo imposible atacando sus causas. La comprensión de que la presencia de los datos en el enunciado, asi como la indicación de todas las condiciones existentes - todo ello como punto de partida--, responde a concepciones inductivistas y orienta incorrectamente la resolución, constituye un paso esencial en el desbloqueo de la enseñanza habitual de problemas y sus limitaciones. Pero al mismo ticmpo genera desconcierto, porque choca con la práctica reiterada, con lo que «siempre» se ha hecho. Un enunciado sin datos, se señala, ¿no será algo excesivamente ambiguo frente a lo cual los alumnos acaben extraviándose? Ahora bien, la ambigüedad $-o$, dicho con otras palabras, las situaciones abiertas - ino son acaso una característica esencial de las situaciones genuinamente problemáticas? ¿Acaso una de las características esenciales del trabajo científico no es la de acotar los probilemas abiertos, imponer condiciones simplificatorias?

Dos dificultades suelen apuntarse durante esta discusión: la primera se refiere a la posibilidad de climinar los datos y precisiones de los cnunciados habituales y construir enunciados más abiertos capaces de generar una resolución acorde con las caracteristicas del trabajo cientifico. A este respecto, el trabajo realizado en numerosos "workshops" y cursos de perfeccionamiento del profesorado, ha permitido consta tar que los enunciados habituales son "traducibles» sin dificultad.

Así, por ejemplo, el enunciado:

«Sobre un móvil de $5000 \mathrm{~kg}$ que se desplaza con una velocidad de $20 \mathrm{~m} / \mathrm{s}$, actúa una fuerza de frenado de $10.000 \mathrm{~N}$, ¿qué velocidad llevará a los $75 \mathrm{~m}$ de donde comenzó a frenar?»

puede ser traducido a una situación más abierta y que no señale cuáles son las magnitudes relevantes, como la siguiente:

«Un automovilista comienza a frenar al ver la luz amarilla, ¿qué velocidad llevará el automóvil al llegar al semáforo?»

Por supuesto, son posibles distintos enunciados, distintas situaciones problemáticas, más o menos abiertas; así, el problema anterior puede dar lugar, entre otros muchos, a este otro enunciado que, aunque aparentemente diferente, plantea una situación muy similar:

«¿Arrollará el tren a la vaca?»

(o «¿Chocará el vehículo con el obstáculo?») 
De hecho, cuando se plantea a varios grupos la traduc. ción de un mismo enunciado tradicional, se obtienen distintas propuestas de situaciones problemáticas, en general igualmente válidas. En cualquier caso, intere* sa destacar que estas transformaciones no plantean dificultades mayores y que cualquier enunciado habitual es transformable en situación problemática (Gil y Mar. tinez Torregrosa, 1987).

Por otra parte, subsiste la cuestión de cómo orientar a los alumnos para abordar dichas situaciones, puesto que no basta, obviamente, con enfrentarles a enuncia. dos sin datos para lograr una actividad exitosa. Se pian. tea, pues, la cuestión fundamental:

¿Qué orientaciones proporcionar a los alumnos para facilitar el tratamiento de situaciones abiertas?

\section{LA RESOLUCIÓN DE PROBLEMAS COMO INVESTIGACIÓN}

La cuestión de qué orientaciones proporcionar a los alumnos para abordar la resolución de problemas sin datos (en las que ya no es posible el simple juego de datos, fórmulas e incógnitas) conduce a los grupos de profesores a propuestas básicamente coincidentes con las que se enuncian a continuación y que, en conjunto, suponen un modelo de resolución de problemas como investigación (Gil y Martínez Torregrosa, 1983):

I. Comenzar por un estudio cualitativo de la situación, intentando acotar y definir de manera precisa el problema, explicitando las condiciones que se consideran reinantes, etc.

Cabe señalar que esto es lo que realizan habituaimente los expertos ante un verdadero problema y lo que en ocasiones se recomienda, sin demasiado éxito. Pero los alumnos, ahora, se ven obligados a realizar dicho análisis cualitativo: no pueden evitarlo lanzándose a operar con datos e incógnitas, porque no disponen de ellos. Han de imaginar necesariamente la situación física, tomar decisiones para acotar dicha situación, explicitar qué es lo que se trata de determinar, ctc. (En el apartado 5.1 incluimos, a modo de ejemplo, el tratamiento completo de una situación problemática).

II. Emitir hipótesis fundadas sobre los factores de los que puede depender la magnitud buscada y sobre la forma de esta dependencia, imaginando, en particular, ca. sos limites de fácil interpretación física,

Asi, por ejemplo, en un problema como «Un automóvil comienza a frenar al ver la luz amarilla, ¿con qué velocidad llegará al paso de peatones? ", no se trata sólo de señalar la infuencia de la fuerza de frenado, masa del automóvil, distancia a la que se encontraba inicial mente del paso de peatones y velocidad que llevaba, sino de predecir la forma de estas relaciones y, repetiv mos, considerar posibies casos límites. Los alumnos siguen así profundizando en la situación física, llegando a plantearse, por ejemplo, que «si la fuerza de frenado fuese nula la velocidad que llevaría seguiria siendo la inicial», etc, etc.

Llamamos la atencion sobre el hecho de que la emisión de hipótesis es quizás la forma más eficaz de conectar con los preconceptos de los alumnos, puesto que expresan en ellas sus ideas intuitivas. $Y$ es preciso recordar que, como toda una abundante investigación ha puesto en evidencia (Posner et Al, 1982; Driver, 1986), no es posible un aprendizaje significativo de los conocimientos científicos si no se plantea dicho aprendizaje como un cambio conceptual, a partir de las ideas intuitivas de los alumnos. Lamentablemente, sin embargo, la invención de hipótesis, que constituye además el núcleo esencial de la metodologia científica, $-\mathrm{y}$, en general, la creatividad-, está ausente de las actividades propuestas a los alumnos en la enseñanza de la resolución de problemas (Gil y Martínez Torregrosa, 1984; Garrett, 1987) como puede constatarse en el anexo I.

III. Elaborar y explicar posibles estrategias de resolución antes de proceder a ésta, evitando el puro ensayo y error. Buscar distintas vías de resolución para posibilitar la contrastación de los resultados obtenidos y mostrar la coherencia del cuerpo de conocimientos de que se dispone.

De nuevo se trata de favorecer aqui el pensamiento divergente, la creatividad consubstancial al trabajo científico y ausente casi por completo de la enseffanza habitual de las ciencias (Yager y Penick, 1983).

IV. Realizar la resolución verbalizando al máximo, fundamentando lo que se hace y evitando, una vez más, operativismos carentes de significación física.

V. Analizar cuidadosamente los resultados a la luz de Ias hipótesis elaboradas y, en particular, de los casos limite considerados.

Este análisis de los resultados puede convertirse en ocasión reiterada de conflicto cognoscitivo, contribuyendo asi al necesario cambio conceptual.

Es conveniente resaltar que las orientaciones precedentes no constituyen un algoritmo que pretenda guiar paso a paso la actividad de los alumnos. Muy al contrario, se trata de indicaciones genéricas destinadas a llamar la atención contra ciertos «vicios metodológicos» con naturales: la tendencia a caer en operativismos ciegos o a pensar en términos de certeza y no de hipótesis, lo que se traduce en no pensar en posibles caminos alternativos de resolución o en no poner en duda y analizar los resultados, etc. Estas orientaciones intentan, pues, ayudar a superar lo que se ha denominado «metodología de la superficialidad" (Gil y Carrascosa, 1985) o "metodologia del sentido común» (Hashweh, 1986), haciendo posible un tratamiento de los problemas a la vez imaginativo y riguroso, acorde con lo que 
constituye la metodología cientifica, sin el cual no es posible concebir ni un enfoque eficaz de los problemas ni la construcción de conocimientos científicos, es decir, el aprendizaje significativo de los mismos.

Expondremos, para terminar, algunos resultados ob. tenidos con alumnos de Enseñanza Secundaria y con profesores en formación y en activo.

\section{IA APLICACIÓN DEL MODELO DE RE- SOLUCIÓN DE PROBLEMAS COMO INVES. TIGACIÓN: ALGUNOS RESULTADOS}

\subsection{Un ejemplo de cómo abordar una situación probtemática}

Vamos a transcribir a continuación, para ejemplificar el modelo de resolución elaborado, el enfrentamiento a una situación problemática, tal como suele desarro. llarse en una clase de enseñanza secundaria.

Consideremos el enunciado:

"¿Chocará el tren con el obstáculo?»

Se trata de la transformación de uno de los enunciados típicos de $2^{\circ}$ de BUP, que presenta el interés -en la forma propuesta aquí- de no explicitar inequivo. camente, como es habitual, qué es lo que se pide. Ello exige, como veremos, un esfuerzo suplementario de reflexión y precisión por parte de los alumnos.

\section{a) Planteamien to cualitativo y formulación de hipótesis}

Las primeras consideraciones cualitativas en las que los alumnos coinciden sin dificultad, conducen a referirse a un tren que avanza con una velocidad determinada y cuyo maquinista, a la vista de un obstáculo, frena para impedir el choque. Según que el problema sea abordado en un contexto cinemático o dinámico, los alumnos traducen el «frena» por «producir una aceleración» o por «aplicar una fuerza». El problema puc. de por supuesto resolverse en cualquiera de los dos niveles. Pero la auténtica dificultad y las discrepancias, aparecen en cuanto se trata de precisar qué es lo que el problema pide. La pregunta «¿chocará...?» no puede traducirse automáticamente a la forma habitual de "calcular la distancia....", o bien "obtener la fuerza...», etc. Y se trata sin embargo de realizar dicha precisión u operativización, sin lo cual dificilmente se podrá seguir avanzando. En otras palabras, los alumnos han de plantearse - y es posible que el profesor necesite impulsarles a elio si por sí mismos no lo hacen- la cues* tion de qué se podría calcular que diera información sobre si el choque se produce o no. Ello conduce a distintos planteamientos que resumimos a continuación:

- Una primera forma de operativizar el problema, es plantearse la obtención de la distancia, d, que recorrerá el tren desde que empieza a frenar (con una fuerza que se considera constante a lo largo de la trayectoria) hasta que se para. Si esa distancia es igual o menor que
D - distancia desde el obstáculo al punto donde se en. contraba el tren cuando empezó a frenar- no chocará.

- Otra forma de abordar el problema propuesta por los alumnos consiste en pensar en términos de velocidad, es decir, calcular la velocidad, $v^{*}$, con que el tren llegará al obstáculo. Si esa velocidad es mayor que ce. ro, el tren chocará.

- Son posibles, además, otras formulaciones del problema, que aparecen con menor frecuencia. Por ejemplo, podría calcularse la fuerza necesaria para que el tren se pare tras recorrer exactamente la distancia D. Si el valor de la fuerza de frenado que puede aplicarse es igual o superior a la obtenida, el choque se evitará.

Para algunos alumnos estos planteamientos pueden parecer distintos, puesto que se trata respectivamente de calcular una distancia, una velocidad y una fucrza. Es fácil comprender sin embargo que ello no es así y que los resultados habrán de ser coincidentes. Precisamente la resolución por más de un procedimiento se convierte en una forma de contrastar la validez de los resultados. En to que sigue, describiremos la resolución realizada según la segunda orientación, es decir, determinando la velocidad que tendrá el tren, al que se aplica una fuerza de frenado constante, cuando liega al obstáculo.

Los alumnos no encuentran dificultad para exponer a modo de hipótesis que la velocidad, $\mathrm{v}^{*}$, dependerá de la velocidad $v_{o}$ que licvaba el tren, de la fuerza de frenado, $F$, de la masa, $m$, del mismo, y de la distancia, $\mathrm{D}$, que recorre el tren desde que empieza a frenar hasta que alcanza el obstáculo.

Algún alumno suele aludir al tiempo que tardará el tren en detenerse, pero, con mayor o menor rapidez, surgen comentarios sobre la dependencia de este tiempo de los mismos factores anteriormente considerados. A este respecto, es necesario advertir a los alumnos la conveniencia de permitir la formulación de cualquier hipótesis sin pasar a criticarlas de entrada, con objeto de evitar efectos inhibitorios. Una vez formuladas todas las hipótesis, es cuando conviene pasar a su discusión y profundización. Asf́, los alumnos expresan que $\mathrm{v}^{*}$ será mayor cuanto mayor sea $\mathrm{v}_{0}, y$ que disminuirá su valor si aumenta el de la fuerza de frenado o/y el de la distancia recorrida hasta alcanzar el obstáculo.

Cuando se separan las variables $\mathrm{F}$ y $\mathrm{m}$ (no utilizando como variable la aceleración), la dependencia de la velocidad con la masa no aparece siempre clara: algunos alumnos afirman que cuanto mayor sea $\mathrm{m}$ el tren disminuirá más rápidamente su velocidad. Esto pone en evidencia una concepción errónea que considera la masa como propiedad que mide la «resistencia» del cuerpo al movimiento, que mediante un razonamiento irreflexivo se convierte en: mayor masa, «más resistencia», luego se parará antes. Otros alumnos, por supuesto, argumentan lo contrario: a mayor masa, con más velocidad llegará, pues la aceleración de frenado será me- 
nor. A pesar de que el argumento es concluyente, con objeto de incidir sobre la concepción errónea citada, se puede dejar en alto esta cuestión y clarificarla a la luz de los resultados obtenidos.

Aún debe irse más allá en la profundización cualitativa e intuitiva en la situación, solicitando a los alumnos que imaginen situaciones límites donde sea posibie avanzar el resultado. Los aluminos se refieren así, a que si el tren no frena $(F=O)$, entonces la velocidad no variará: $\mathrm{v}^{*}=\mathrm{v}_{0}$; lo mismo ocurriría si el conductor no advirtiera el obstáculo hasta encontrarse junto a él, es decir, si $\mathrm{D} \rightarrow \mathrm{O}$, será $\mathrm{v}^{*}=v_{\text {o. }}$. Cabe esperar también que si la masa del tren es muy grande ( $m$ $\rightarrow \infty$ ), la fuerza de frenado no afecte a la velocidad, de nuevo entonces, $v^{*}=v_{0}$. Strelen indicar, del mismo modo, que si la fuerza de frenado es "infinita", la velocidad con que llegará el tren al obstáculo será nula. Esta última intervención abre un debate en el que se clarifica que no es necesario que la fuerza tienda a infinito para que la velocidad, $v^{*}$, sea cero. Por el contrario, queda claro que existe un valor finito de $F$ que hará que $\mathrm{y}^{*}$ sea cero, es decir, que el tren se pare justo al llegar al obstáculo. Si $F$ supera dicho valor, el tren simplemente se parará antes, o lo que es lo mismo, no tendrá sentido preguntar el valor de $v$ al llegar al objeto.

Queremos insistir, por una parte, en el hecho de que son las hipótesis, a la luz de la visión cualitativa del problema, las que determinan lo que debe considerarse «datos» necesarios para su resolución (en este caso, vo, F, m y D) en contra del error empirista, favorecido por el tipo habitual de enunciado, consistente en tomar los datos como punto de partida. Por otra parte, esta formulación de hipótesis ha de entenderse como algo más que la simple enumeración de factores: la consideración de posibles casos límite obliga precisamente a profundizar más en dichas hipótesis, ayudando a predecir resultados concretos, con claro significado físico, que facilitarán después el análisis de resultados.

En definitiva, pues, el planteamiento cualitativo inicial, la formulación fundamentada de hipótesis y el avance de casos límites como profundización en las mismas, permiten así abordar de los problemas como tentativas que ponen en juego las estructuras conceptuales y la imaginación creativa de los alumnos, convirtiendo el resto del problema en un proceso para verificar si el planteamiento inicial o las hipótesis concebidas eran o no adecuadas.

Es ahora el momento de proponer posibles estrategias de resolución.

\section{b) Fstrategias de resolución y resolución propiamente dicha}

Las estrategias concebidas por los alumnos dependen obviamente del cuerpo de conocimientos de que disponen. Una primera estrategia propuesta consiste en el tratamiento cinemático/dinámico del movimiento del tren -o, para ser más precisos, de la cabecera del tren- suponiendo un movimiento uniformemente acelerado. Si los alumnos han estudiado ya las relaciones trabajo/energía, proponen también considerar la variación de energía cinética que provoca el trabajo de frenado.

No vamos aqui a transcribir más detalladamente las estrategias propuestas y los procesos de resolución que se derivan, y que conducen al resultado:

$\mathrm{v}^{*}=\sqrt{\mathrm{v}_{\mathrm{o}}^{2}-2 . \mathrm{F} . \mathrm{D} / \mathrm{m}}$. Pero sí insistiremos en que esta búsqueda de estrategias representa para los alumnos un proceso complejo para el que no disponen de "reglas infalibles», -como algunos intentos de algoritmización pretenden-, si bien puede resultar muy útil el análisis en partes diferenciadas, el establecimiento de analogías con situaciones ya tratadas, el estudio previo de casos más simples, etc. En general se trata de una tarea en la que, por su propia naturaleza, no quedan excluidas, claro está, las rectificaciones o el replanteamiento global, que las aleja de una simple caplicación de conocimientos». Es precisamente este carácter de enigma, como decíamos más arriba, el que es olvidado en la didáctica habitual de los problemas, en la que las estrategias suelen aparecer como algo seguro de antemano, como frutos de una intuicion inmediata sólo al alcance del profesor. Por el contrario, con el modelo propuesto los alumnos son conscientes -y lo aceptan como algo natural- de que las situaciones de incertidumbre son inherentes al abordaje de problemas, es decir, a los procesos de investigación.

\section{c) Análisis de los resultados}

El paralelismo establecido entre resolución de un problema y proceso de investigación permite comprender la importancia que ha de concederse -en contra de la práctica habitual- al análisis de los resultados. En efecto, dicho análisis ha de jugar el papel de constrastación de las hipótesis emitidas, permitiendo averiguar hasta qué punto la visión cualitativa de la situación (origen de todo el desarrollo) era correcta y/o la estrategia seguida adecuada, etc.

En primer lugar, los alumnos se refieren a la obtención del mismo resultado con los dos procedimientos de resolución tutilizados. Por otra parte, señalan que, tal como avanzaban en sus hipotesis, la velocidad con que el tren llega al obstáculo depende de la velocidad inicial, aumentando o disminuyendo si esta lo hace; que cuanto mayor sea la fuerza de frenado o/y la distancia a la que se encuentra inicialmente el tren del obstáculo, $\mathrm{D}$, menor será la velocidad $\mathrm{v}^{*}$, etc., etc.

Con respecto a la masa, la expresión obtenida indica que la velocidad final será mayor al aumentar la mis. ma, lo que contradice conjeturas iniciales que son de nuevo discutidas, contribuyendo a una clarificación/profundización de los conocimientos. Los alumnos pueden también verificar los casos límites avanza- 
đos; así, por ejemplo, si $F=0$, es decir si el tren no frena, $\mathrm{v}^{*}=\mathrm{v}_{\mathrm{o}}$.

De manera que el resultado obtenido para $v^{*}$ parece lógico y puede ser aceptado como correcto por los alumnos (que pueden además comprobar su homogeneidad dimensional).

La respuesta a la pregunta planteada por el problema, se obtiene concluyendo que si $v^{*}>0$ el tren chocará con cl obstáculo. Es decir, si $\left(\mathrm{vo}_{0}^{2}-2 \mathrm{~F} \mathrm{D} / \mathrm{m}\right)>0$, el tren chocará, y si $\mathrm{v}_{0}^{2}=2 \mathrm{~F} \mathrm{D} / \mathrm{m}\left(\mathrm{v}^{*}=\mathrm{O}\right)$, se detendrá justo al llegar al obstáculo.

A partir de esta expresión es posible obtener distintas interpretaciones coincidentes. Por ejemplo, chocará si la fuerza de frenado no alcanza un determinado valor, concretamente, si $\mathbf{F}<\mathrm{m} \mathrm{v}_{0}^{2} / 2 \mathrm{D}$. De otro modo, el tren no podrá evitar chocar con el obstáculo si la distancia a la que empieza a frenar es menor que un valor determinado: $\mathrm{D}<\mathrm{m} \mathrm{vo} 2 / 2 \mathrm{~F}$.

Por supuesto, alcanzado este punto, los alumnos pueden solicitar al profesor los datos que precisen para obtener un valor numérico. En ocasiones es conveniente que ellos mismos imaginen los datos con objeto de que se habitúen al manejo de valores plausibles.

\subsection{Cambios metodológicos y actitudinales}

Hemos mostrado hasta aquí que es posible crear condiciones que permitan al profesorado poner en cuestión su tratamiento didáctico de los problemas y llevarles a (re)construir un modelo de resolución como investigación, acorde con las características básicas del trabajo científico. Y hemos transcrito, a título de ejemplo, el tratamiento de una situación problemática abierta, desde el planteamiento inicial al análisis de los resultados, tal como pueden realizarlo grupos de alumnos en su clase, y también grupos de profesores durante un curso de puesta en cuestión de la didáctica habitual como el que hemos descrito.

El número de problemas que es posible abordar con la nueva metodología en las sesiones de trabajo de uno de estos cursos no es nunca elevado, pero, en todo caso es suficiente para que los profesores tengan los elementos necesarios para valorar comparativa. mente el nuevo modelo y la didáctica habitual. A continuación presentamos los resultados obtenidos con un elevado número de profesores de enseñanza secundaria en activo (162) y en formación (80) (Tablas 1 y 2).

Tabla I

Valoración comparativa por los profesores de las metodologías de resolución y presentación didáctica de los problemas.

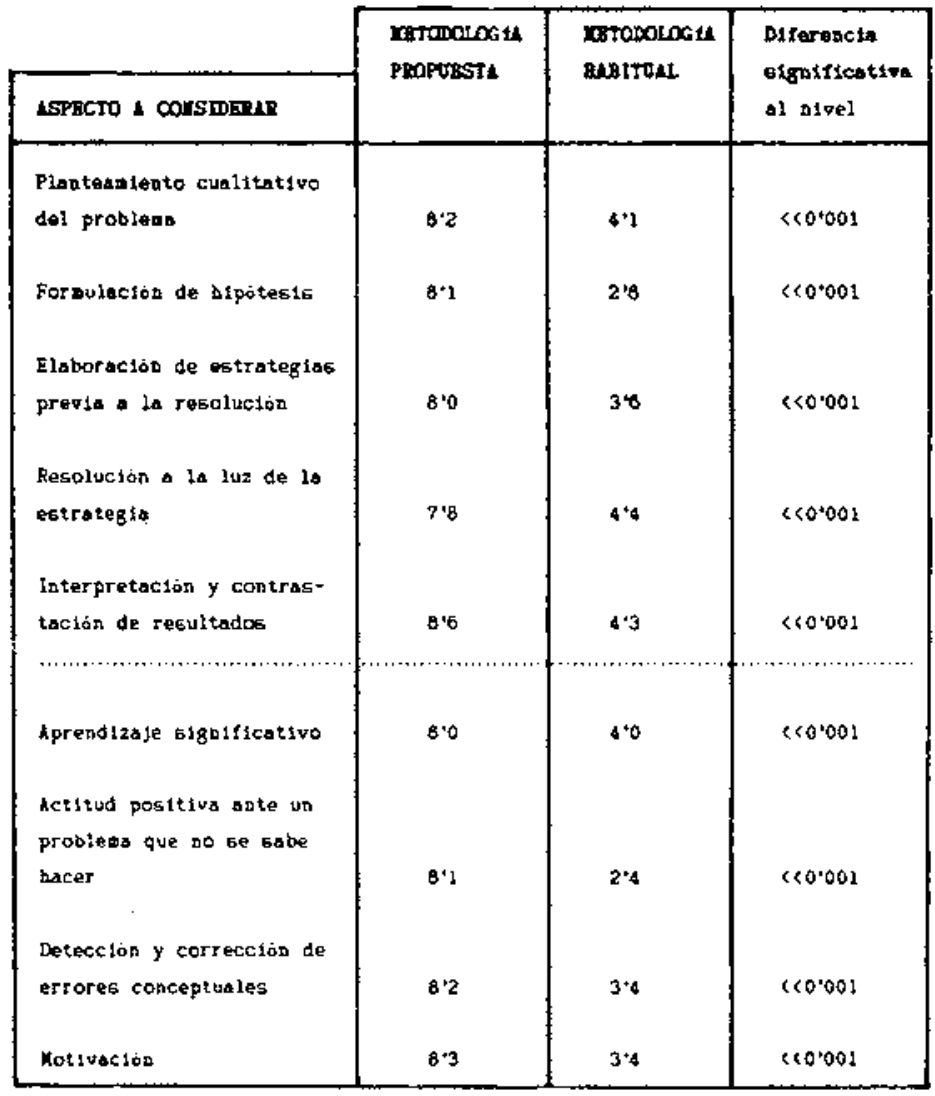


Tabla i]

Valoración comparativa por los profesores en formación (alumnos de los C.A.P.) de las metodologias de resolución y presentación đidáctica de los problemas.

\begin{tabular}{|c|c|c|c|}
\hline ISFECTO I COISTEBRAI & $\begin{array}{l}\text { Frmatoris } \\
\text { ropotsta }\end{array}$ & $\begin{array}{l}\text { IRTODOLOFith } \\
\text { HABITULL }\end{array}$ & $\begin{array}{l}\text { Diferacte } \\
\text { sigolficativa } \\
\text { al nivel }\end{array}$ \\
\hline $\begin{array}{l}\text { Planteos lento cuslitative } \\
\text { del probiane }\end{array}$ & $8 \%$ & 45 & $<\left(0^{\prime} 001\right.$ \\
\hline $\begin{array}{l}\text { Formulactiós de bipotesis } \\
\text { Elaboración de estrategias } \\
\text { previs a ta racoluclion }\end{array}$ & $8 \times 8$ & $2 ' 2$ & $<<0<001$ \\
\hline $\begin{array}{l}\text { Resolución a la luz de la } \\
\text { estrategis }\end{array}$ & 8.7 & 10 & $<<0.001$ \\
\hline $\begin{array}{l}\text { laterpretación y contras- } \\
\text { tactos de resultados }\end{array}$ & 96 & $3 \cdot 3$ & $<<0,001$ \\
\hline Aprendiafe signilleativo & 816 & $3 ' 6$ & $<<0,001$ \\
\hline $\begin{array}{l}\text { Actitud positiva ante un } \\
\text { problewa que no se sabe } \\
\text { dacer }\end{array}$ & B.9 & $2 ' 2$ & $<<0.001$ \\
\hline $\begin{array}{l}\text { Deteccion y correcclion de } \\
\text { errores conceptuales }\end{array}$ & $9 \cdot 1$ & $2 " ?$ & $<(0,00)$ \\
\hline Xotjuaction & 8.6 & 322 & $<<0,001$ \\
\hline
\end{tabular}

Resaltan de inmediato las notables diferencias entre las valoraciones de ambas metodologías. No sólo consideran que con el modelo propuesto se favorece la familiarización de los alumnos con aspectos básicos de la metodologia científica - tales como planteamientos cualitativos, formulación de hipótesis..., análisis de resultados- sino que sus expectativas respecto a las posibilidades de la resolución de problemas para favorecer un aprendizaje significativo, crear una actitud positiva ante un problema que no se sabe hacer, su utilidad para detectar y corregir errores conceptuales y conseguir motivar a los alumnos, se ven aumentadas en más de un $100 \%$.

En resumen, estas valoraciones muestran claramente no sólo que el modelo de resolución como investigación es altamente valorado respecto a la metodología habitual, sino, también, que produce una motivación en los propios profesores, un cambio en sus actitudes y expectativas que resulta imprescindible para el «cambio didáctico».

A pesar de ello, es lógico que aparezcan dudas sobre la validez y aplicabilidad del modelo en las clases, por lo que es necesario presentar aquí algunos resultados obtenidos con alumnos.
Ya hemos mostrado, en el ejemplo del apartado 5.1, cómo el uso sistemático del modelo en la clase acerca la actividad de los alumnos a las características del trabajo científico, evitando el operativismo y la «linealidad" que caracterizaría la presentación didáctica habitual de la resolución de problemas. Esta orientación fomenta el uso de formas de pensamiento cualitativas y abiertas, y a la vez, de modo indisoluble, analíticas, rigurosas y precisas, contribuyendo así a la superacion de la «metodología de la superficialidad». No obstante es necesario comprobar que, efectivamente, la práctica reiterada del modelo produce este cambio metodológico en los alumnos, aumenta su capacidad para resolver problemas y modifica su actitud e interés hacia esta actividad. De un modo operativo, esto significa que (Gil y Mtnez. Torregrosa, 1986):

1) Los alumnos tratados no caigan en la manipulación inmediata de datos y fórmulas, típica del operativismo ciego. Por el contrario, cabe esperar que

2) dediquen un tiempo inicial a las consideraciones cualitativas, formulaciones precisas del problema, etc.,

3) formulen hipótesis fundamentadas, operativizándolas e imaginando posibles casos limites con una significación física clara, 
4) expliciten posibles estrategias de resolución antes de iniciarla, intentando buscar más de un camino para resolver el problema...,

5) realicen una resolución con explicaciones que den sentido a lo que se hace,

6) analicen los resultados obtenidos, considerando las hipótesis avanzadas, la verificación de los casos límites...

Además de las consecuencias que acabamos de enumerar, que responden, en buena medida, a lo que solicita el modelo explícitamente, vamos a referimos tambièn a otras consecuencias que mostrarian indirectamente la validez del modelo para provocar el cambio metodológico: en efecto, esperamos que la utilización reiterada del modelo produzca:

7) una mayor capacidad para resolver problemas, lo que se traducirá en que los alumnos tratados obtendrán un porcentaje de resultados correctos notable y significativamente superior al de alumnos no tratados.

8) Cabe esperar también, que dediquen más tiempo a intentar la resolución de un problema que les resulte dif́icil antes de abandonar, sin caer, por tanto, en la actitud habitual de "reconocer o abandonar" (Gilbert, 1980) típica de la metodología de la superficialidad.

Por último, en to que se refiere a la modificación de las actitudes de alumnos hacia esta actividad esencial para el aprendizaje de las ciencias, cabe esperar que:

9) la práctica del modelo aumente de modo notable y duradero el interés de los alumnos por la resolución de problemas, a causa de la naturaleza más abierta y creativa de la orientación propuesta, y también a causa de los mejores resultados que hace posible.

El desarrollo detallado del diseño experimental para la contrastación de estas características en las resoluciones de los alumnos puede encontrarse en Gil y Mtnez. Torregrosa (1986) y, más detalladamente, en Mtnez. Torregrosa (1987). Sólo indicaremos aquí que, aunque se ha empleado el modelo durante varios cursos académicos con estudiantes de $2^{\circ}$ y $3^{\circ}$ de BUP y de COU, los resultados que se presentan corresponden a un grupo experimental de $3^{\circ}$ de BUP, utilizando como grupos de control uno de $3^{\circ}$ de BUP y dos grupos de COU. Para comparar el comportamiento de estos grupos, hemos utilizado tres problemas con las caracteristicas siguientes:

Los problemas han sido presentados en la forma habitual, es decir, con datos numéricos, tal como suelen aparecer en los libros de texto.

Dos de los enunciados contenían datos superfluos con el fin de poder detectar su posible manejo operativista por parte de los alumnos. Debemos precisar que ninguno de los grupos se habia enfrentado antes a enunciados con exceso de datos.
Uno de los problemas se ha pasado a los grupos de $3^{\circ}$ de BUP en situación de examen.

El conjunto de resultados obtenidos muestra diferencias importantes y estadísticamente significativas entre el grupo experimental y los de control en todos los aspectos medidos. Resumiremos brevemente estos resultados:

$\left.1^{\circ}\right)$ La Tabla 3 muestra la utilización inmediata de los datos como un a priori, en los grupos de control. Esta utilización inmediata de los datos, sin reflexionar sobre su pertinencia, es todavía más evidente en la Tabla 4 , donde puede observarse el elevado porcentaje de estudiantes de estos grupos que hacen uso de datos superfluos. Estos resultados - que pueden considerarse expresión de lo que es habitual en la resolución de problemas- contrastan clara y significativamente ( $p<0$ 01 en todos los casos) con los que muestran el comportamiento del grupo experimental.

\section{Tabla III}

Los datos aparecen inmediatamente.

\begin{tabular}{|c|c|c|c|}
\hline & $\begin{array}{l}\text { CRUPO } \\
\text { EXPERIMERTAL } \\
\text { (30 LP) }\end{array}$ & 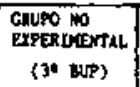 & $\begin{array}{l}\text { GRTIFO NO } \\
\text { EXPEADSINTA. } \\
\text { (COU) }\end{array}$ \\
\hline PROBLOXU HABITJAL & $\begin{array}{cc}2 & 56 \\
\text { is } & \left(6^{\circ}, 9\right\}\end{array}$ & $\begin{array}{cc}z & \text { sd } \\
100 & (-)\end{array}$ & $\begin{array}{cc}x & 5 \\
89^{\prime} 7(3 \cdot 7)\end{array}$ \\
\hline 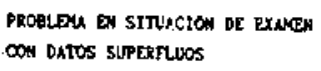 & $179\left(0^{+} 1\right)$ & $971\left(2^{\prime} 1\right)$ & $\rightarrow \cdots$ \\
\hline 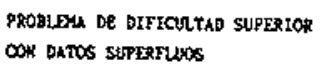 & is (I's) & $100(-)$ & $100(-)$ \\
\hline
\end{tabular}

Tabia IV

Los alumnos utilizan datos superfluos.

\begin{tabular}{|c|c|c|c|}
\hline & $\begin{array}{l}\text { CRUPO } \\
\text { EXPEh tKLATAL } \\
\left(J^{\circ} \text { BLP }\right)\end{array}$ & $\begin{array}{l}\text { GRUPO WO } \\
\text { EXPE IMENTAL } \\
\text { (J० QAP) }\end{array}$ & $\begin{array}{l}\text { CAAPO KO } \\
\text { SXPER INEKTAL } \\
\text { (COU) }\end{array}$ \\
\hline $\begin{array}{l}\text { PROBLDU EN SITURCTON DE EXNIEN } \\
\text { CON DXIOS SUPEKRUUOS }\end{array}$ & $\begin{array}{cc}t & 54 \\
10 \cdot 3 & (4.9)\end{array}$ & $\begin{array}{cc}2 & 5 d \\
54^{\prime} 4 & \left(6^{\prime} 0\right)\end{array}$ & $\begin{array}{cc}2 & \text { Sa } \\
-2 & \cdots\end{array}$ \\
\hline $\begin{array}{l}\text { PROALDAR DE DIFICULTAD SUPERTOF } \\
\text { CON BATOS SUPERFWOS }\end{array}$ & $22.5(6.6)$. & $52.0\left(10^{\circ} 0\right)$ & $35+0(6.8)$ \\
\hline
\end{tabular}

En lo que respecta al momento de la introducción de las formulas -otro indicador indudable del operativismo habitual - las Gráficas 1 y 2 muestran también notables diferencias entre el grupo experimental y los de control, haciendo evidente el cambio producido por la aplicación del modelo.

$\left.2^{\circ}\right)$ Las diferencias son igualmente marcadas y signi- 
Gráfica 1

Momento en que aparecen las fórmulas en ia resolución del problema habitual.

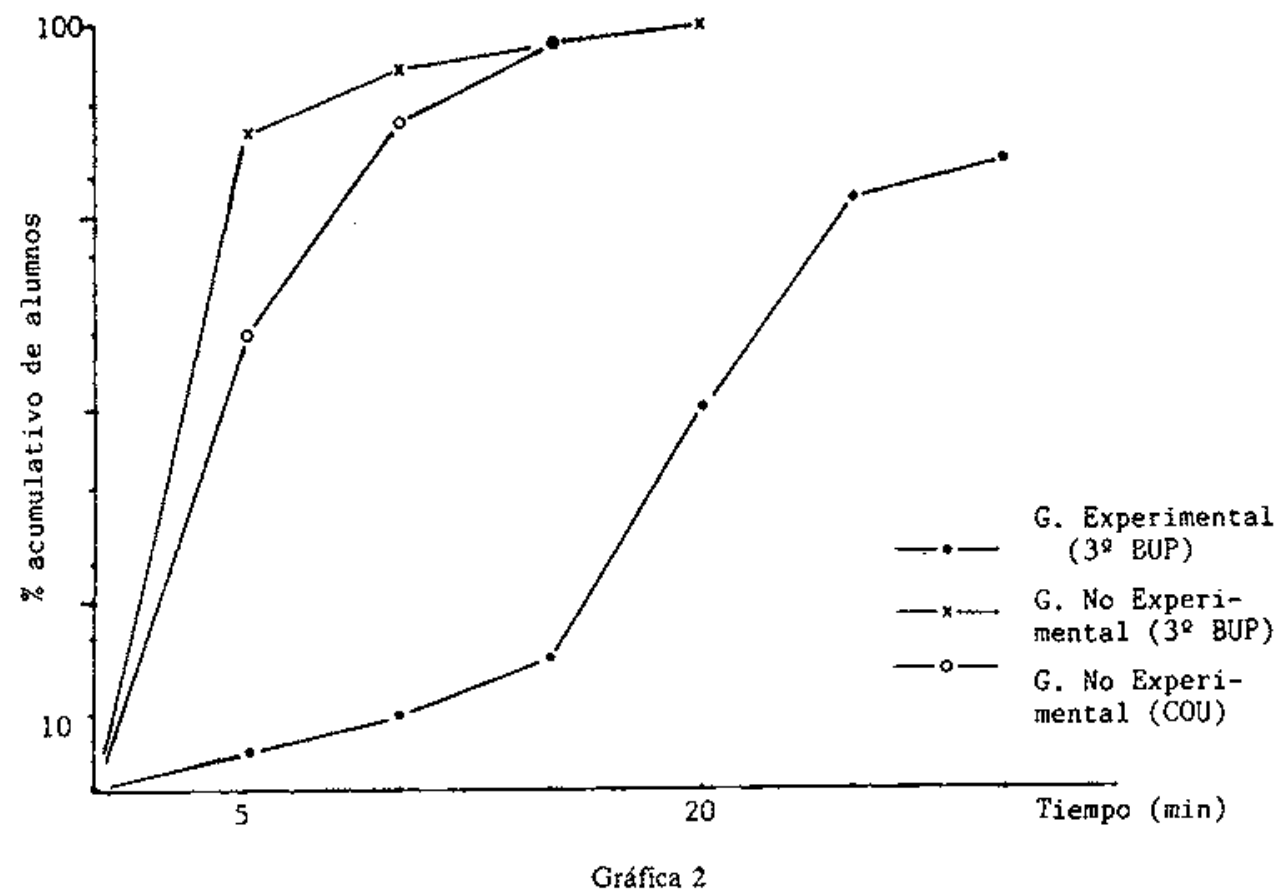

Momento en que aparecen las fórmulas en la resolución del problema de dificulaad superior con datos superfluos.

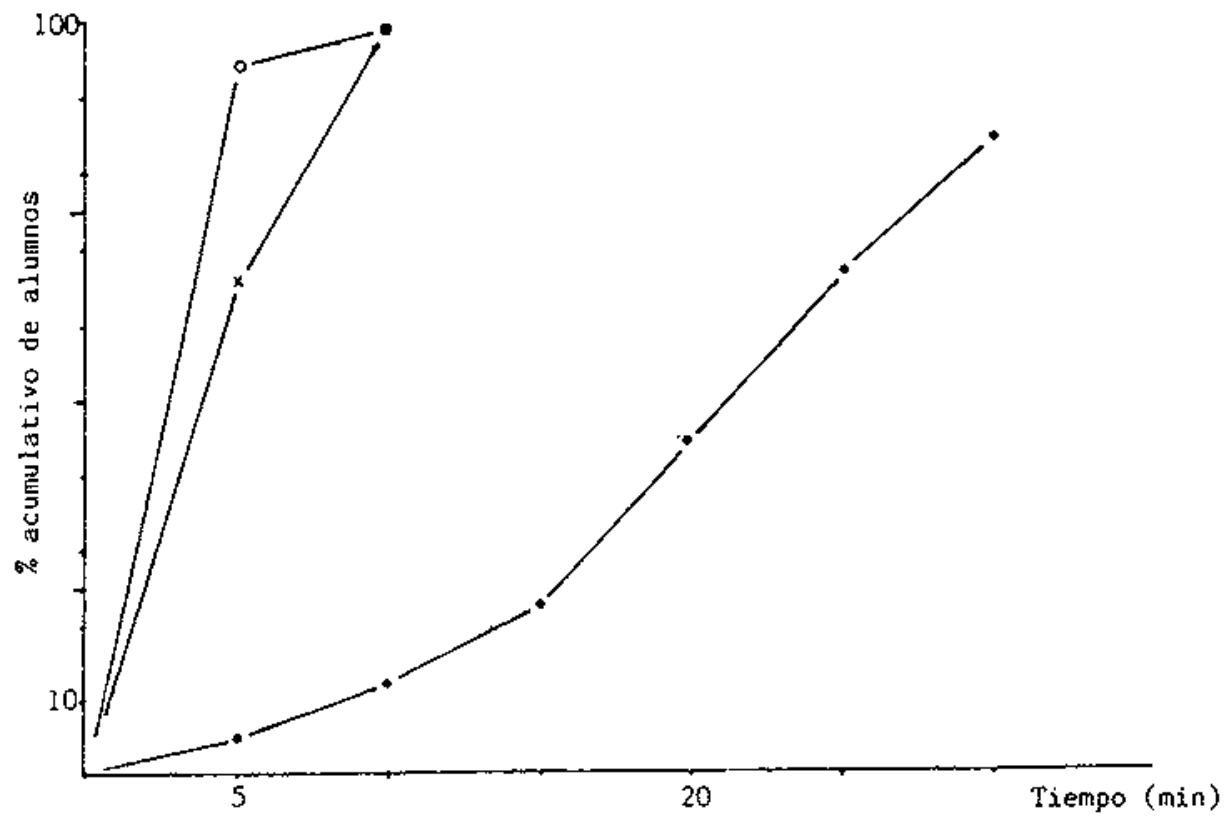

ficativas en lo que se refiere a todos los aspectos clave del trabajo cientifico: desde la existencia o no de planteamientos cualitativos iniciales, la formulación de hipótesis..., hasta el análisis de los resultados.

A modo de ejemplo, hemos incluido las Tablas 5 y 6 que se refieren respectivamente a la explicitación o no de las estrategias antes de proceder a la resolución y a la existencia o no de análisis de los resultados. Las diferencias que muestran estas tablas hacen innecesario todo comentario. Debe resaltarse, además, que se trata de dos actividades que se consideran necesarias por parte de todos los autores, independientemente de su orientación; las diferencias son, incluso, más mar- 
cadas cuando se consideran otras actividades como la emisión de hipótesis y el uso de casos limites, etc.

$\left.3^{\circ}\right)$ Nos referiremos ahora a tres aspectos especialmente relevantes y claramente relacionados entre sí y con el cambio metodológico. Se trata de la calidad de la

Tabla $V$

Se elabora una estrategia untes de empezar fa resolucjón.

\begin{tabular}{|c|c|c|c|c|c|c|}
\hline \multirow[b]{2}{*}{ PROBCEHA HABITUAL } & \multicolumn{2}{|c|}{$\begin{array}{l}\text { CRUPO } \\
\text { EXPEAYKERTAL } \\
(10 \text { BUP })\end{array}$} & \multicolumn{2}{|c|}{$\begin{array}{c}\text { CRUPO NO } \\
\text { EXPERTMEKTAL } \\
(3 * \text { BLPP })\end{array}$} & \multicolumn{2}{|c|}{$\begin{array}{l}\text { CRUPO NO } \\
\text { EXPERIXENTAL } \\
(\text { OOU) }\end{array}$} \\
\hline & & $\begin{array}{l}56 \\
\left(6^{\prime} 3\right)\end{array}$ & 10.5 & $\left(5^{\prime} 0\right)$ & 73 & $(3 \cdot 2)$ \\
\hline $\begin{array}{l}\text { PHOBLEHA EN SITUACIOH DE EXAMEN } \\
\text { CON EXCESS DE DATOS }\end{array}$ & $14 \% 6$ & $\left(7^{\prime} 0\right)$ & 0 & $(-)$ & - & -- \\
\hline $\begin{array}{l}\text { PSOBLEYA DE DIFICURTAB SUPERIOR } \\
\text { ON DATOS SUFERFLUS }\end{array}$ & 52.5 & (7'9) & $3 \prime 1$ & $\left(3^{\prime} 1\right)$ & 0 & $(-)$ \\
\hline
\end{tabular}

Tabla VI

Se analiza el resutado.

\begin{tabular}{|c|c|c|c|c|c|c|}
\hline \multirow[b]{2}{*}{ PROBLEYA HABITUAL, } & \multicolumn{2}{|c|}{$\begin{array}{l}\text { CRUPO } \\
\text { ExpER]MEXTAL } \\
\left(33^{2} \text { BuP }\right)\end{array}$} & \multicolumn{2}{|c|}{$\begin{array}{l}\text { GRUPO WO } \\
\text { EXPRPIMENTAL } \\
\text { (jo WUP) }\end{array}$} & \multicolumn{2}{|c|}{$\begin{array}{l}\text { CRUPO NO } \\
\text { EXPER!NENTLL } \\
(O Q N)\end{array}$} \\
\hline & & $\begin{array}{l}56 \\
(7+5)\end{array}$ & 2.6 & $\left(2^{4} 6\right)$ & 29 & $\left(2^{\prime} 0\right)$ \\
\hline $\begin{array}{l}\text { PROALEYA EN SITUKCLON DE EXNMEM } \\
\text { CON EXCESO OE OATOS }\end{array}$ & 716 & $\left(J^{\prime} 2\right)$ & & $(-)$ & $-\cdot$ & - \\
\hline $\begin{array}{l}\text { PROBL,DU OE OIFICURTAD SUPERTON } \\
\text { CON DATOS SUPERFLUOS }\end{array}$ & 35 & $(7.9)$ & 0 & $(-)$ & 0 & $(-)$ \\
\hline
\end{tabular}

resolución (Tabla 7), de los porcentajes de alumnos que abandonan (Tabla 8) y de los tiempos destinados a la resolución de un problema (Gráfica 3). La Tabla 7 no sólo muestra que los estudiantes del grupo experimen. tal obtienen sistemáticamente mejores resultados en los tres problemas, sino que las diferencias aumentan con
Tabla VII

El resultado obtenido es correcto.

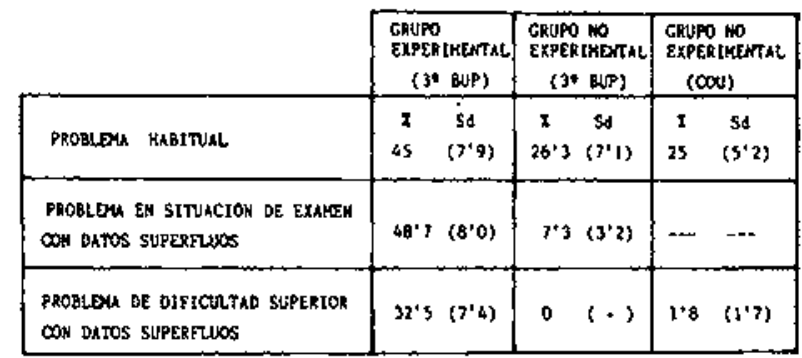

Tabla Vifi

Porcentaje de alumnos que abandonan.

\begin{tabular}{|c|c|c|c|}
\hline & $\begin{array}{l}\text { CRUPO } \\
\text { EXPERIMENTAL. } \\
\text { (3* aUP). }\end{array}$ & $\begin{array}{c}\text { GRLPO WO } \\
\text { EXPER IMENTAL } \\
\text { (30 BUP) }\end{array}$ & $\begin{array}{l}\text { CRUPO NO } \\
\text { EXPER IMENT AL } \\
\text { (COU) }\end{array}$ \\
\hline PROBLEH HABITUKL & $\begin{array}{c}1 S^{\prime} 5\left(5^{\prime} 2\right) \\
\left.12^{\prime}\right)\end{array}$ & $\begin{array}{cc}1 & 5 d \\
26 \cdot 3 & (7 \cdot 1)\end{array}$ & $\begin{array}{cc}x & 54 \\
30.9 & (5.6)\end{array}$ \\
\hline $\begin{array}{l}\text { PROBLDUK EN SITUACIOK DE EXNLIN } \\
\text { COK DATOS SUPERRUOS }\end{array}$ & $2+6$ & $177(6.6)$ & $\cdots$ \\
\hline $\begin{array}{l}\text { PROBLOMA DE OIFICULTAO SUPEQTOH } \\
\text { CON DATOS SUPERTUNOS }\end{array}$ & $2+5 \quad(2 \cdot 5)$ & $68^{\prime}+\left(8^{\prime} 2\right)$ & $35 \times(6.3)$ \\
\hline
\end{tabular}

Gráfica 3

Tiempo total utilizado en ta resolución del problema de dificultad superior con datos superfluos.

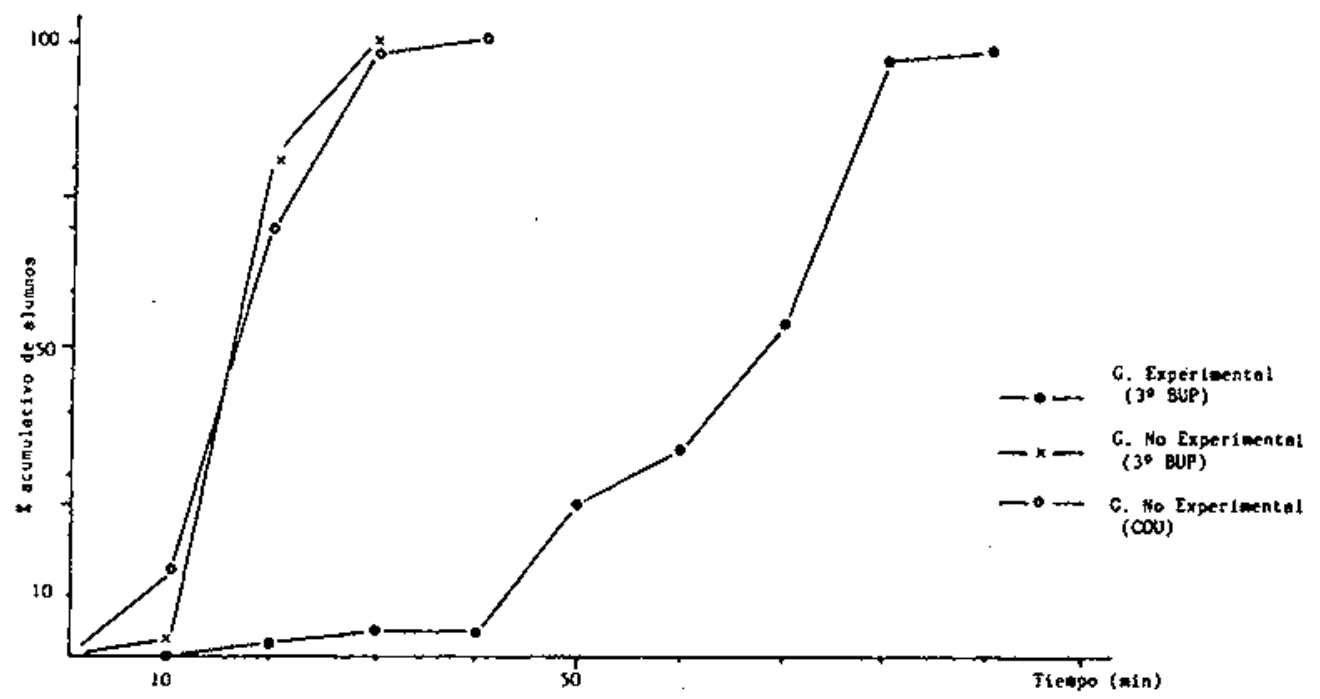


la diftcultad del problema. Se hace evidente, de este modo, hasta qué punto no se enseña a resolver problemas sino a memorizar resoluciones explicadas por el profesor, lo que hace que los alumnos se encuentren literaimente perdidos cuando no reconocen el "problema» (Gil y Mtnez. Torregrosa, 1984). Esto se hace todavía más evidente cuando se considera el número de alumnos que abandonan la resolución (Tabla 8) o el tiempo que se destina a resolver el problema. En la Gráfica se muestra cómo - a pesar de poder disponer de todo el tiempo necesario- casi un $50 \%$ de los alumnos no tratados había acabado antes de los 20 minutos y un $98 \%$ a los 30 minutos (con sólo un 7,3\% de resultados correctos). Se muestra también con claridad, por el contrario, la mayor perseverancia y tenacidad adquiridas por los estudiantes tratados, lo que es una manifestación más de un indudable cambio metodológico. En la tabla 9 se presenta el número medio de frases por alumno, como un índice del grado de verbalización (tan importante en la resolución de problemas) y de la ucalidad" de la resolución.

Tabla IX

Grado de verbalización (numero medio de frases/alumno).

\begin{tabular}{|c|c|c|c|}
\hline & 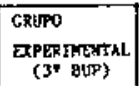 & 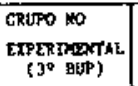 & $\begin{array}{l}\text { GREPO HO } \\
\text { EXPERDERTTLL } \\
\text { (COU) }\end{array}$ \\
\hline PROBLDAA HABTRUAL & נ' 16 & $3 \cdot 3$ & $5+0$ \\
\hline $\begin{array}{l}\text { PROALDU EN SITHACION DE ERNAEN } \\
\text { COK EXCESO DE OATOS }\end{array}$ & ו'23 & 122 & -- \\
\hline $\begin{array}{l}\text { PROELDU DE DIFICULTAD SUPERIOR } \\
\text { CON DATOS SUPEAFUNOS }\end{array}$ & 2216 & $2^{\prime} 4$ & $0 * 2$ \\
\hline
\end{tabular}

$4^{\circ}$ ) Por último, en la Tabla 10, se recoge la valoración que han hecho los alumnos del grupo experimental sobre el modelo, nueve meses después de haber finalizado el curso, cuando se encuentran ya utilizando durante seis meses la orientación didáctica habitual para la resolucion de problemas. Se pone en evidencia asi, que el cambio metodológico que favorece el modelo está ligado - de acuerdo con nuestra hipotesis - a un verdadero cambio actitudinal, resultado del carácter abierto y creativo de la forma de resolución propuesta y de la autoconfianza y mejores resultados que posibilita.

\section{CONCLUSIONES}

La investigación sobre la resolución de problemas se ha orientado, durante decenios, a establecer las diferencias entre «buenos» y «mediocres» resolventes y a diseñar algoritmos de resolución para facilitar la tarea de los alumnos. Hemos intentado justificar por qué estas líneas de investigación se han mostrado inefectivas, explicitando y analizando críticamente los supuestos subyacentes en las mismas. $\mathrm{Y}$ hemos concebido nuevas vías de aproximación a partir de un cuestionamiento en profundidad del tipo de enunciados propuesto (muy alejados de lo que constituye una auténtica situación problemática) y de las orientaciones didácticas utilizadas para su resolución (consistente en la presentación lineal, "desproblematizada» de "la solución»).

Frente a ello, hemos intentado mostrar que la traducción de los enunciados habituales en situaciones abiertas ( $\sin$ los habituales datos y especificación de las condiciones existentes) es susceptible de generar actividades caracteristicas de un tratamiento científico de los problemas y, muy en particular, sus aspectos más creativos. Se favorece asi, no sólo una drástica reducción del fracaso en la resolución de problemas, sino un auténtico cambio metodológico y actitudes mucho más positivas en profesores y alumnos.

Tabla X

Valoración comparativa de las actitudes e intereses que generan las distintas metodologias de resolución de problemas utilizadas por alumnos tratados. $(\mathrm{N}=30)$

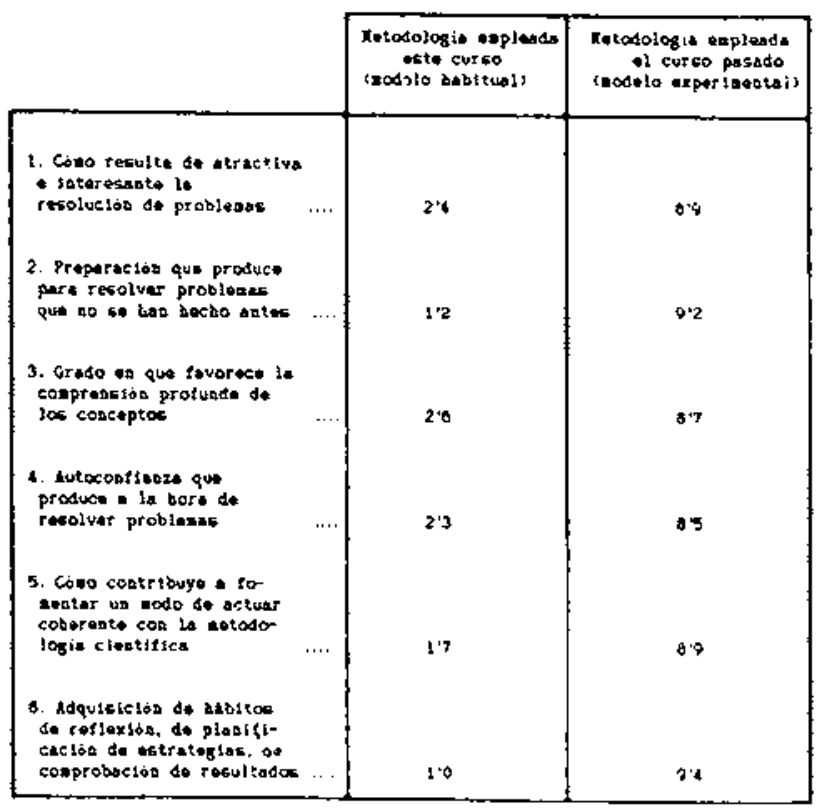




\section{REFERENCIAS BIBLIOGRÁFICAS}

BODNER, G.M. y McMILLEN, T.L.B., 1986, Cognitive restructuring as an early stage in problem solving, Journal of Research in Science Teaching, 23 (8), 727-737.

BROMME, R., 1984, On the linitations of the theory methaphor for the study of teacher's expert knowledge, en Halkes R. Olson J.K. (eds), Teachers thinking. $A$ new perspective on pesisting problems in education. (Swets Zeitlinger: Lisse, NL).

DUMAS-CARRE, A., 1987, Résolution de problèmes en physique au lycée. Tesis de Estado presentada en la Universidad de París VII.

DRIVER, R., 1986, Psicología cognoscitiva y esquemas conceptuales de los alumnos, Enseñanza de las Ciencias, 4 (1), 3-15.

GARRETT, R.M., 1986, Problem solving and creativity in science education, Estudies in Science Education, 13, 70-95.

GARRETT, R.M., 1987, Issues in science education: problem-solving, creativity and originality, International Journal of Science Education, vol. I, pp 26.33.

GIL, D., 1983, Tres paradigmas básicos en la enseñanza de las ciencias, Enseñanza de las Ciencias, vol. 1, pp 26-33.

GIL, D., 1986, La metodologia cientifica y la enseñanza de las ciencias: unas relaciones controvertidas, Enseñanza de las Ciencias 4 (2), 111-121.

GIL, D. y CARRASCOSA, J., 1985, Science learning as a conceptual and methodological change, European Journal of Science Education, vol. 7, $\mathrm{n}^{\circ} 3,231+236$.

GIL, D. y MTNEZ. TORREGROSA, J., 1983, A model for problem solving in accordance with scientific methodology, European Journal of Science Education, vol 5, pp 447-455.

GIL, D. y MTNEZ. TORREGROSA, J., 1984, ProblemSolving in Physics: a critical analysis. Research on Physics Education (Editions du CNRS: Paris),

GIL, D. y MTNEZ. TORREGROSA, J., 1986, La resolución de problemas como instnumento de cambio metodo. lógico, Educación Abierta, n 66, pp 31-59.

GIL, D. y MTNEZ. TORREGROSA, J., 1987, La resolución de problemas de Física (Ediciones del M.E.C.: Madrid y Vicens Vives: Barcelona).

GIL, D., MTNEZ. TORREGROSA, J. y SENENT, F,, $1987 \mathrm{~b}, \mathrm{LA}$ resolution de problemes comme instrument de changement conceptuel et methodologique, Petit $X, n^{\circ}$ 14-15, pp. 25*38.

HASHWEH, M.Z., 1986, Towards an explanation of conceptual change, European Journal of Science Education 8 (3), 229-249.

HAYES, J.R., 1981, The complete problem solver (The Franklin Insitute Press: Philadelphia).

HODSON, D., 1985, Philosophy of science, science and science education, Studies in Science Education, 12, 25-57.

HUDGINS, B.B., 1966, Cómo enseñar a resolver problemas en el aula (Paidós: Buenos Aires).

LARKIN, J.H. y REIF, F., 1979, Understanding and teaching problem solving in Physics. European Journal of Science Education, I (2), 191.203.

MAHAPATRA, J.K., 1987, Can problem+solving in physics give an indication of pupils "process knowledge»?, International Journal of Science Education, 9 (1), 117-123.

MARTÍNEZ TORREGROSA, J., 1987, La resolución de problemas como investigación: un instrumento de cambio metodológico. Impublished PH. D. Thesis. Servei de publicacions de la Universitat de València.

METTES et AJ, 1980, Teaching and learning problem solving in science. Part I: a general strategy. Journal of Chemical Education, 57, 882-885.

MILLAR, R. y DRIVER, R., 1987, Benyond processes, Studies in Science Education, 14, 33-62.

PIAGET, J., 1974, Introduction a l'Epistemologie Génétique II. La pensée Physique. (P.U.F.: París).

POLYA, G., 1957, How to solve it (Dobleday Co.: Garden City, N.Y.).

POSNER, G.I., STRIKE, K.A., HEWSON, P.W. y GERTZOG, W.A., 1982, Accomodation of a scientific conceptions: towards a theory of conceptual change, Science Education, 66, 211-227.

RIBAS, M., 1986, Factores de eficacia escolar: una linea de investigación didáctica, Bordón, 264, 693-708.

RICHE, N., 1978, Trame pour rédiger un exercise de physique, Revue Française de Pédagogie, 45, 183-199.

YAGER, R.E. y PENICK, J.E., 1983, Analysis of the current problems with school science in the USA, European Journal of Science Education, 61, 463-469. 


\section{ANEXO \\ ALGUNOS RESULTADOS SOBRE EL ANÁLISIS DE LA PRESENTACIÓN DIDÁCTICA HABITUAL DE LA RESOLUCIÓN DE PROBLEMAS.}

Tabla XI

Resultados de análisis de las caussas de fracaso en la resolución de problemas citadas por profesores y profesores en formación.

Xuestra : 55 profesores de Bachllerato en ejercicto; 122 profesorac en foranción de Bachillerato (C.A.P.) y 91 profesores en formaclón de Magtaterlo (de 22 y 32 de lo espectalldad de Ciencias).

\begin{tabular}{|c|c|c|c|c|c|c|}
\hline \multirow{2}{*}{ 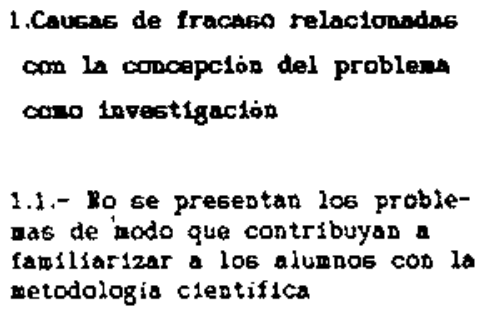 } & \multicolumn{2}{|c|}{ Profecores } & \multicolumn{2}{|c|}{$\begin{array}{c}\text { Profesores on } \\
\text { Pormacion } \\
\text { (C.L.P.) }\end{array}$} & \multicolumn{2}{|c|}{$\begin{array}{l}\text { Prolecores en } \\
\text { Formación } \\
\text { Oagleter 1o) }\end{array}$} \\
\hline & 55 & $(3)$ & $\mathbf{z}$ & $\left(11^{\prime} 6\right)$ & * & $(19)$ \\
\hline $\begin{array}{l}\text { 1.2.- Presedtactos lineal por } \\
\text { parte del profesor }\end{array}$ & $3 \cdot 6$ & $(2 ' 5)$ & 6 & $(2 \cdot 1)$ & 3 & $(219)$ \\
\hline $\begin{array}{l}\text { 1.3.- So se favorece el pensamien- } \\
\text { to divergente }\end{array}$ & 0 & $(-1)$ & 0 & 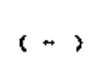 & 0 & $(-)$ \\
\hline $\begin{array}{l}\text { 1.4.- Do se elabora una estrategia } \\
\text { antes de la resoluction }\end{array}$ & 0 & $(-)$ & 0 & $(*)$ & 1 & $(-)$ \\
\hline $\begin{array}{l}\text { 1.5.- Yo se enseta al alumno } \\
\text { analizar los resultados }\end{array}$ & $3^{\prime} 6$ & $(2 ' 5)$ & 0 & $(-)$ & 0 & $(-)$ \\
\hline $\begin{array}{l}\text { 1.6.- Otras causas que se puedat } \\
\text { relacionar con dtcha concepció }\end{array}$ & $7 \cdot 3$ & $(3 \cdot 5)$ & 9 & $\left\{2^{*} 6\right\}$ & 8 & $(28)$ \\
\hline $\begin{array}{l}\text { 2. Lectura no comprensiva del } \\
\text { edunclado }\end{array}$ & 47 & $(6+7)$ & 30 & $(4)$ & 38 & $\left(5^{\prime} 1\right)$ \\
\hline 3. Deflcteate preparacfós teórica & 40 & $(6 \cdot 6)$ & 48 & $\langle 4 \cdot 5\rangle$ & 48 & $\langle 5 ` 2\rangle$ \\
\hline $\begin{array}{l}\text { 4. Deficlente manefa del aparato } \\
\text { antenatico }\end{array}$ & 38 & $(6 \cdot 5)$ & 26 & $(4)$ & 6 & $(2 \cdot 4)$ \\
\hline
\end{tabular}


Tabia XII

Resultados del análisis de los problemas resueltos por profesores y profesores en formación

Muestra: 55 profesores de Bachillerato en ejercicio; 122 profesores en formación de Bachillerato (C.A.P.) y

91 profesores en formación de Magisterio (de $2^{\circ}$ y $3^{\circ}$ de la especialidad de Ciencias)

Muestra: 63 LIBROS

15 de EGB,

23 de BUP,

10 de COU,

8 de Física General y

7 de problemas resuettos.

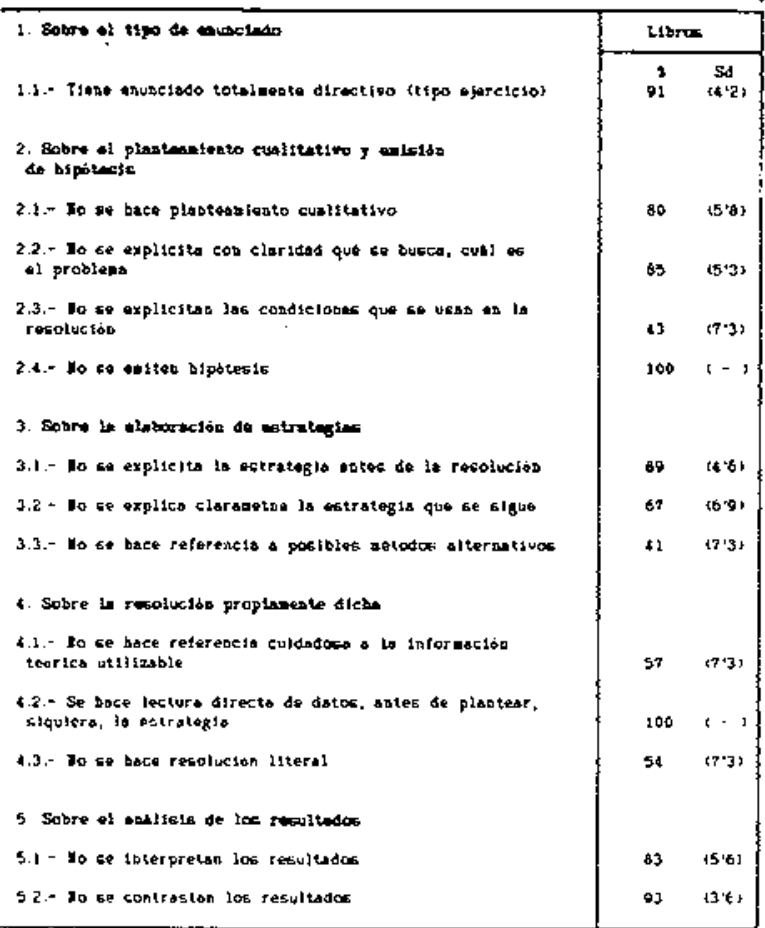

Tabla XIII

Resultados del análisis de libros de texto y de problemas

\begin{tabular}{|c|c|c|c|c|c|c|}
\hline \multirow{2}{*}{ 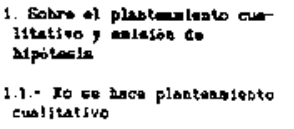 } & \multicolumn{2}{|c|}{ 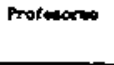 } & \multicolumn{2}{|c|}{ 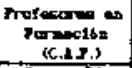 } & \multicolumn{2}{|c|}{ 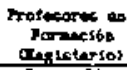 } \\
\hline & & $\begin{array}{l}S \alpha \\
15,03\end{array}$ & & $\begin{array}{l}56 \\
13 \cdot 8)\end{array}$ & 60 & Sd \\
\hline 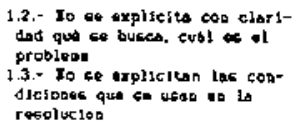 & of & $(4,5)$ & 78 & $\begin{array}{l}\{3,4\} \\
\{3,8\}\end{array}$ & 90 & $\begin{array}{l}\{2 \cdot 6\} \\
\{2 ' 2\}\end{array}$ \\
\hline 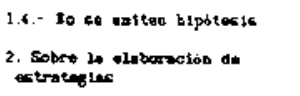 & 100 & 21 & 100 & $\cdots$ & 200 & , \\
\hline 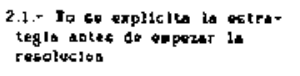 & 84 & $(5.4)$ & 26 & (3') & 100 & $1-9$ \\
\hline 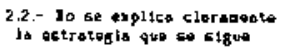 & 67 & $(5 \cdot 3)$ & & $\{3 \cdot 6\}$ & 99 & $(1)$ \\
\hline 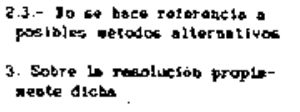 & 80 & 4546 & 83 & $22^{\prime 4}$ & 100 & $t-3$ \\
\hline 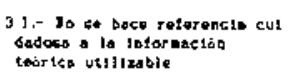 & 64 & 1653 & 68 & $(12)$ & 9 & $13 \cdot 31$ \\
\hline $\begin{array}{l}\text { 3a.- No se bace rezoluetion } \\
\text { Jileral }\end{array}$ & & 16041 & 06 & $\{3\}$, & 80 & +13 \\
\hline 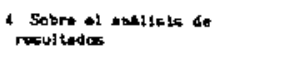 & & & & & & \\
\hline $\begin{array}{l}\text { d- No se inte-pretas los } \\
\text { resuitodas }\end{array}$ & 78 & $15 \cdot 63\}$ & 81 & $: 353$ & 98 & $11+52$ \\
\hline $\begin{array}{l}\text { 6.2.- Mo se contractos } 106 \\
\text { resultados }\end{array}$ & 67 & $(4.5)]$ & 90 & $\left(2^{\prime} 7\right\}$ & 80 & $(15)$ \\
\hline
\end{tabular}

